Frédéric Dumont*, Hélène Senellart, Francois Pein, Loic Campion, Olivier Glehen, Diane Goere, Marc Pocard and Emilie Thibaudeau

\title{
Phase I/II study of oxaliplatin dose escalation via a laparoscopic approach using pressurized aerosol intraperitoneal chemotherapy (PIPOX trial) for nonresectable peritoneal metastases of digestive cancers (stomach, small bowel and colorectal): Rationale and design
}

https://doi.org/10.1515/pap-2018-0120

Received June 12, 2018; accepted August 22, 2018;

previously published online September 15, 2018

\section{Abstract}

Background: The annual incidence of gastrointestinal carcinomas (stomach, small bowel, colon and rectum) is increasing in Western countries, reaching 50,000 new cases each year in France. Peritoneal carcinomatosis (PC) is diagnosed in $15 \%$ of these patients. Complete cytoreductive surgery (CCS) plus Hyperthermic IntraPeritoneal Chemotherapy (HIPEC) is the only therapy that can offer patients with PC a chance for long-term survival with a 5 year overall survival (OS) rate of 30$60 \%$ versus $0-5 \%$ with systemic chemotherapy alone. However, CCS plus HIPEC still presents serious limitations and very few patients $(10 \%)$ are candidates for these radical treatments. PC remains a palliative setting for $90 \%$ of patients with a median survival ranging from 15 to 25 months. Innovative surgical therapies such as Pressurized IntraPeritoneal Aerosol Chemotherapy (PIPAC) therefore need to be developed to improve the prognosis. Potential benefits were obtained after intraperitoneal nebulization of oxaliplatin in patients with

*Corresponding author: Frédéric Dumont, Department of Surgical Oncology, Institut Cancérologique de l'Ouest, Saint Herblain, France, E-mail: frederic.dumont@ico.unicancer.fr Hélène Senellart, Department of Medical Oncology, Institut Cancérologique de l'Ouest, Saint Herblain, France Francois Pein, Department of Clinical Research and Innovation, Institut Cancérologique de l'Ouest, Saint Herblain, France Loic Campion, Department of Biostatistics and Methodology, Institut Cancérologique de l'Ouest, Saint Herblain, France

Olivier Glehen, Department of Surgical Oncology, Centre Hospitalier Lyon Sud, Pierre Bénite, France

Diane Goere, Department of Surgical Oncology, Gustave Roussy Cancer Campus, Villejuif, France

Marc Pocard, Department of Digestive and Oncological Surgery, Lariboisière Hospital, AP-HP, Paris 7-Diderot University, Paris, France

Emilie Thibaudeau, Department of Surgical Oncology, Institut Cancérologique de l’Ouest, Saint Herblain, France advanced PC from colorectal cancer. Innovative surgical therapies such as pressurized intraperitoneal aerosol chemotherapy (PIPAC) have been proposed as palliative locoregional treatment with some promising results. The dose of oxaliplatin currently established by nebulization (PIPAC) is really low at $92 \mathrm{mg} / \mathrm{m}^{2}$. However, the peritoneum acts as a barrier limiting the systemic passage of intraperitoneal drug. Oxaliplatin used at higher doses during PIPAC procedures could be a safe option and allow better intratumoral penetration of chemotherapy.

Method and design: The proposed study is a multicenter phase I/II trial of oxaliplatin dose escalation during PIPAC. The aim is to determine the maximum tolerated dose of pressurized oxaliplatin administered by the intraperitoneal route (PIPAC) during two consecutive procedures at a 4-6 week interval for patients with extended peritoneal carcinomatosis from the gastrointestinal tract. Dose started at $90 \mathrm{mg} / \mathrm{m}^{2}$ and escalation was in $50 \mathrm{mg} / \mathrm{m}^{2}$ steps up to a maximum of $300 \mathrm{mg} / \mathrm{m}^{2}$.

Discussion: Oxaliplatin is an effective drug in gastrointestinal cancer and high doses given by the intraperitoneal route during HIPEC are well tolerated. In this phase I trial, we hypothesized that high-dose oxaliplatin during PIPAC is feasible and safe. The repeated local administration of high doses of oxaliplatin could improve tumor response and prognosis.

Trial registration: Prospective study. ClinicalTrials.gov: NCT03294252. EudraCT: 2016-003666-49

Keywords: colorectal carcinoma, gastrointestinal carcinoma, oxaliplatin, peritoneal carcinomatosis, PIPAC

\section{Background}

The annual incidence of colorectal and gastric carcinomas is increasing in Western countries [1], with 40,000 and 7,000 new cases, respectively, each year in France [2]. Peritoneal carcinomatosis (PC) is diagnosed in $15 \%$ of these patients $[3,4]$. Complete cytoreductive surgery 
(CCS) plus Hyperthermic IntraPeritoneal Chemotherapy (HIPEC) is the only therapy that can offer patients with $\mathrm{PC}$ a chance for long-term survival with a 5 year overall survival (OS) rate of $30-60 \%$ [5-9] versus $0-5 \%$ with systemic chemotherapy alone $[8,9,10,11]$. CCS plus HIPEC as well as systemic chemotherapy present serious limitations.

\section{The limitations of systemic chemotherapy}

The benefit of systemic chemotherapy remains limited in case of PC from gastrointestinal carcinoma. In two prospective randomized studies including 2095 patients with colorectal metastases and treated with systemic chemotherapy only, the prognosis of PC patients was poor. [10] Despite the use of modern chemotherapy combining five-FU, irinotecan and oxaliplatin in healthy adult patients, the median OS was still only 15 months with very little prolonged survival $(4.1 \%$ at 5 years). The improvement in survival rates conferred by targeted therapies for metastatic colorectal cancers is far less evident in PC metastases. In a register study among a non-selected population [12], the median survival in case of colorectal PC was 7.5 months and the use of bevacizumab led to only an 11 month median survival. PC from gastric or small bowel carcinomas is considered a terminal stage with a very poor prognosis ranging from 4 to 13 months $[13,14]$.

\section{The limitations of cytoreductive surgery plus HIPEC}

The first limitation of CCS plus HIPEC is high morbidity and mortality. Among 24 studies concerning 2787 patients treated with CCS plus HIPEC [15], the mean mortality was $2.9 \%$ ( $0 \%$ to $17 \%$ ) and major morbidity was $28.8 \%$ ( 0 to 52\%). In a French multicenter study [16] including 1290 patients, the mortality rate was $4 \%$ and $34 \%$ of patients' experienced major morbidity (NCI-CTCAE grade $3 / 4$ ).

Moreover, CCS plus HIPEC does not offer any survival benefit over palliative surgery for extensive PC. Peritoneal extension is evaluated by the Peritoneal Cancer Index (PCI) [17]. For colorectal PC, the cutoff value of PCI is 17 . The median survival rate for patients with PCI higher than 17 is low and close to that obtained with current systemic chemotherapy alone [18]. These results do not justify the morbidity and mortality risks inherent to this procedure [18]. In the case of PC from gastric carcinoma, the PCI score cutoff is around $7[5,19]$. The 5 year OS after CCS plus HIPEC for a PCI between 06, 7-12 and 13-19, was $30 \%, 0 \%$ and $0 \%$, respectively [19]. For small bowel carcinoma, the PCI score cutoff is 15 [8]. For a PCI score $<15$, the median OS at 5 years was $65 \%$ while a median survival of 12 months was observed in case of $\mathrm{PCI}>15$.

Repeat CCS plus HIPEC is rarely performed because of adhesions and poor prognosis. This is another limitation of this procedure. Only $7 \%$ to $12 \%$ of patients with intra-abdominal recurrences receive repeat CCS plus HIPEC $[16,20]$.

Due to the many inherent limitations of CCS plus HIPEC, very few patients (10\%) radical treatments of PC from gastric, bowel or colorectal carcinomas and most $(90 \%)$ are treated with systemic palliative chemotherapy.

\section{Principle and results of pressurized intraperitoneal aerosol chemotherapy (PIPAC)}

PIPAC combines the benefits of a minimally invasive approach with low morbidity and easy to repeat with the pharmacokinetic advantages of intraperitoneal administration. Moreover, the hyperpressure of laparoscopy facilitates better tumor penetration of cytotoxic drugs [21] and aerosol delivery allows a good spatial diffusion of chemotherapy [22]. Demtröder reported 17 patients in good general condition [23] with extended PC (mean PCI score =16) from colorectal carcinoma at time of relapse following a modern systemic chemotherapy (FOLFOX or FOLFIRI, and/or Cetuximab, and/or Bevacizumab): 48 PIPAC procedures were performed using oxaliplatin at a mean dose of $92 \mathrm{mg} / \mathrm{m}^{2}$. Out of 14 patients who received at least two PIPAC procedures, complete pathological response was observed in 50\% (7/14) and 4/14 patients experienced a major pathological response (29\%). In this context (second line treatment), a 15 month median survival is encouraging. Nadiradze reported patients with extended PC (mean PCI score=16) from gastric carcinoma, treated in third or fourth line by systemic chemotherapy in $46 \%$ of cases [24]. Despite frequent resistance to platinum-based systemic chemotherapy, PIPAC still used a platinum salt (cisplatin) in combination with doxorubicin to avoid the local acquired resistances to platinum salts. Most patients (12/24; 50\%) experienced a pathological objective response. The 
preliminary clinical experience with PIPAC is encouraging, although it is still a palliative measure and not in itself a radical treatment of PC as in the case of complete cytoreductive surgery.

The intraperitoneal pharmocokinetics of oxaliplatin during HIPEC are well-known. High intraperitoneal doses (five-fold higher than the systemic dose) of oxaliplatin in 5\% dextrose solution are well tolerated and facilitate tumor concentration [25]. However, the tolerance and pharmacokinetics of high-dose oxaliplatin given by intraperitoneal pressurized aerosol are unknown.

\section{Methods and design}

The PIPOX study is a multicenter, noncomparative, nonrandomized phase I-II trial. This investigator-initiated trial will be conducted by the Department of Surgery of the Institut Cancérologique de l'Ouest (Saint-Herblain, France) in collaboration with the University Hospital Lyon Sud (Pierre Bénite, France), Gustave Roussy Institute (Villejuif, France) and University Hospital Lariboisière (Paris).

\section{Study objectives and endpoints}

The primary objective of the PIPOX study in patients with extended or nonresectable PC arising from digestive carcinoma, is to determine the maximum tolerated dose (MTD) of pressurized oxaliplatin administered by the intraperitoneal route (PIPAC) and the recommended dose for Phase-II during two consecutive procedures performed 4 to 6 weeks apart.
The secondary endpoints are to evaluate:

In the phase I part:

- the types of dose limiting toxicities (DLT) and the toxicity profile of two PIPAC procedures according to the surgical morbidity classification (Clavien-Dindo) [26] and the NCI-CTCAE toxicity scale version 4 [27].

- pharmacokinetics of oxaliplatin aerosol by the intraperitoneal route.

In the phase II part:

- overall tolerance after a total of three to five procedures.

- tumor response rate.

- overall and progression-free survival.

- secondary resection rate.

\section{Statistical considerations}

Toxicity monitoring: DLT is defined as any severe chemotherapyrelated toxicity (grade $\geq 3$ according NCI-CTCAE v4) or unexplained post-operative complication. The recommended dose will be the MTD which is defined as the dose level immediately below the level at which two DLTs were observed.

An independent monitoring committee can be requested if a severe toxicity or complications present a particular difficulty of analysis or if a doubt on the benefit of the study is present.

Statistical analysis plan: The Phase-I part of the study will consist of oxaliplatin dose escalation according to a modified Fibonacci sequence " $3+3$ " design (Figure 1), starting with the dose currently used in PIPAC procedures $\left(90 \mathrm{mg} / \mathrm{m}^{2}\right)$. Dose escalation was in $50 \mathrm{mg} /$ $\mathrm{m}^{2}$ steps up to a maximum of $300 \mathrm{mg} / \mathrm{m}^{2}$, which is the dose currently used in HIPEC procedure. Before moving to the next dose level,

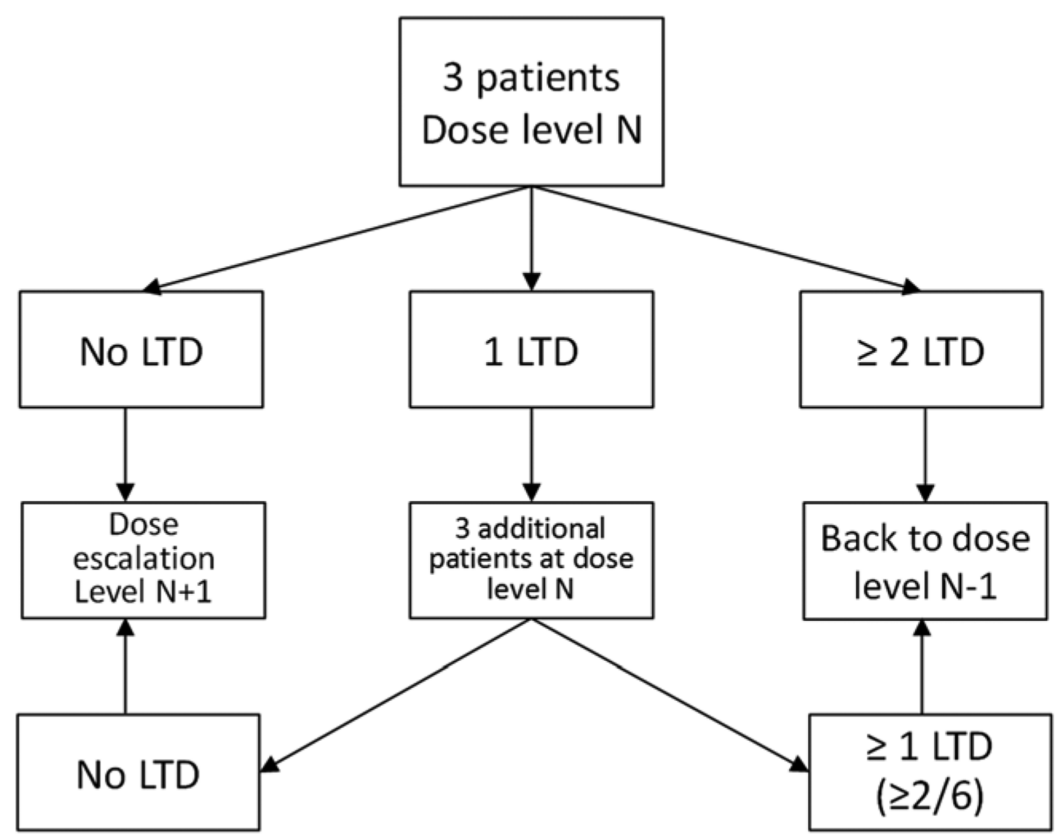

Figure 1: Dose escalation according to a " $3+3$ " Fibonacci sequence. 
at least three patients have to be treated at each level without presenting any severe chemotherapy-related toxicity or unexplained post-operative complication. Each patient who experiences good tolerance (and no tumor progression) will be invited to receive two to five PIPAC procedures at 4-6 week intervals, to allow an evaluation of cumulative toxicities. Therefore, the DLT evaluation period will extend from the start of the first PIPAC procedure up to 4-6 weeks after the second PIPAC procedure, i.e. during an 8-12 week period for a given patient treated at a given dose level.

In the phase-II part of the multicenter study, at the same determined dose level, all the 20 additional patients will be evaluated in terms of safety, histologic response, survival and rate of secondary CCS followed by HIPEC during 3 years of follow-up.

\section{Study population and eligibility criteria}

\section{Inclusion criteria:}

- Patient aged $\geq 18$ years with no upper age limit

- Peritoneal carcinomatosis histologically or cytologically proven

- At least 3 months of systemic chemotherapy

- Extended or nonresectable peritoneal carcinomatosis

- Extended peritoneal carcinomatosis from gastric cancer (PCI >5)

- Extended peritoneal carcinomatosis from small bowel cancer (PCI $>13)$

- Extended peritoneal carcinomatosis from colorectal cancer (PCI $>15)$

- $\quad$ Good general health status - ECOG Performance status $(E C O G \leq 2)$

- Life expectancy $>3$ months

- Peripheral neuropathy grade $\leq 1$

- Hematology: hemoglobin $\geq 9 \mathrm{~g} / \mathrm{dL}$, leukocytes $\geq 4000 / \mathrm{mm}^{3}$, $\mathrm{PMN} \geq 1500 / \mathrm{mm}^{3}$, platelets $\geq 100,000 / \mathrm{mm}^{3}$

- Normal renal function: serum creatinine $\leq 2$ times upper limit of normal (ULN)

- Normal hepatic function: total bilirubin $\leq 1.5 \times$ ULN, ASAT and ALAT $\leq 3 \times$ ULN, alkaline phosphatases $\leq 3 \times$ ULN

- Effective contraception in non-menopausal women

- Signed informed consent

- Patient affiliated with a health insurance scheme in the EU

\section{Exclusion criteria:}

- Last bevacizumab infusion within the last 2 months

- Prolonged corticosteroid treatment, not stopped within 3 months before PIPAC

- Extra-peritoneal metastases with the exception of fewer than 3 pulmonary nodules $<5 \mathrm{~mm}$ in size

- Known hypersensitivity to oxaliplatin

- Complete DPD enzyme deficiency

- Peripheral neuropathy grade $>1$

- Active uncontrolled infection or any uncontrolled severe disease

- Any other cancer with the exception of skin basal cell carcinoma or in situ cervical carcinoma

- Pregnancy or breast-feeding women

- Patient deprived of liberty or under guardianship

- Follow-up impossible due to any reason (geographical, social, psychological, ... .)

\section{Treatment schedule (Figure 2)}

Operative technique: One hour before PIPAC we deliver systemic intravenous leucovorin $20 \mathrm{mg} / \mathrm{m}^{2}$ and $5-\mathrm{FU} 400 \mathrm{mg} / \mathrm{m}^{2}$. All procedures are performed in compliance with Occupational Health and Safety requirements: closed abdomen, laminar airflow, controlled aerosol waste, protection curtain, specific check list.

After insufflation of $12 \mathrm{mmHg}$ of capnoperitoneum, three balloon trocars are placed. Exploratory laparoscopy is performed as usual and the PCI score is determined. Parietal biopsies are taken before and after nebulization for histologic response assessment and translational studies. A nebulizer is connected to a high-pressure injector and inserted into the abdomen through a trocar. A pressurized aerosol containing oxaliplatin at a dose of 90 to $300 \mathrm{mg} / \mathrm{m}^{2}$ body surface in $150 \mathrm{~mL}$ of $5 \%$ dextrose solution is applied. Then, the system is kept in steady-state for $30 \mathrm{~min}$ (application time). Toxic aerosol is exhausted over a closed system. Trocars are retracted.

\section{Translational studies}

Pharmacokinetics and tissue distribution study: The goal of pharmacokinetics study is to determine the physiology of toxicities and therapeutic effectiveness.

The time course of oxaliplatin in the peripheral blood, the tissue distribution and tissue concentration of oxaliplatin will be analyzed. Platinum determination will be performed by inductively coupled plasma mass spectrometry.

At each PIPAC session, five plasma, two peritoneal fluid and three tissue samples are collected.

Plasma samples are collected just before PIPAC, at $10 \mathrm{~min}, 30$ min, $1 \mathrm{~h}, 3 \mathrm{~h}, 6 \mathrm{~h}, 24 \mathrm{~h}$ and $48 \mathrm{~h}$ after the end of each PIPAC procedure. A peritoneal fluid is collected before and after oxaliplatine nebulization for each PIPAC session. At the end of each PIPAC procedure three types of solid tissue will be studied: one tumor nodule, one piece of normal peritoneal tissue treated by PIPAC and one piece of parietal muscle no treated by PIPAC.

Epimarks mutations study: At each PIPAC session, one plasma and two tissue samples are collected. This translational project is designed to provide a first version of a biomarkers-associated schedule for the dynamic combination of oxaliplatin with chemo- and immunomodulatory therapies for the treatment of peritoneal metastasis from colorectal cancer.

\section{Quality assurance}

The protocol will be conducted in compliance with Good Clinical Practice guidelines and the ethical principles set forth in the Declaration of Helsinki. The study protocol was approved by the leading Ethics Committee and approved by the national competent authority (ANSM, ref: 160880-A12) considered mandatory by federal law. The study was assigned the EudraCT number 2016-003666-49 and is registered at ClinicalTrials.gov (NCT03294252). 


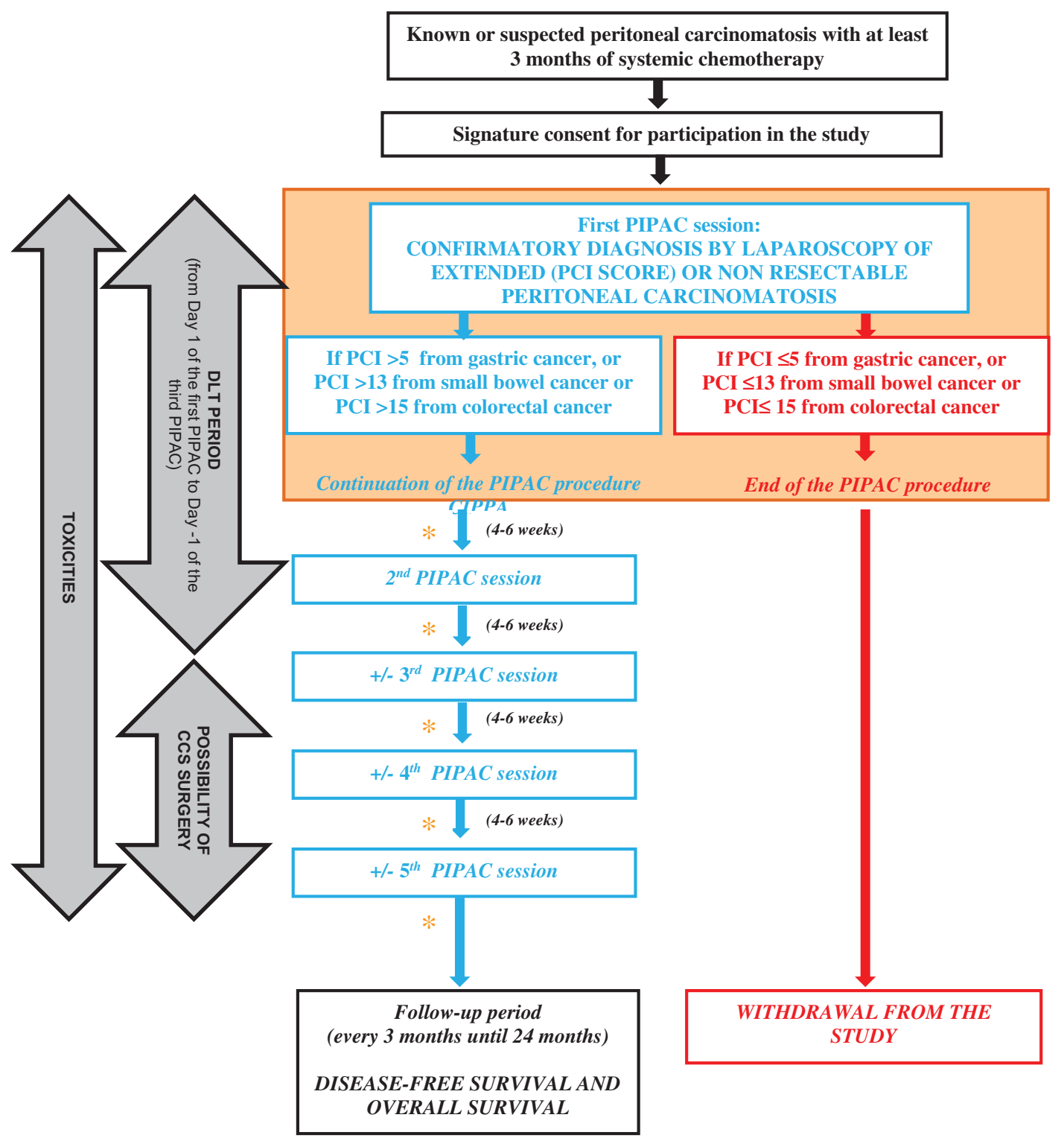

* \pm systemic chemotherapy allowed 2-3 weeks after each PIPAC

Figure 2: Treatment schedule.

\section{Discussion}

PIPOX is the first trial involving oxaliplatin dose escalation via a PIPAC approach. The first patient was included in June 2017. In this phase I trial, we hypothesized that high doses of oxaliplatin during repeated PIPAC are feasible and safe. Oxaliplatin is the most interesting drug to evaluate in PIPAC procedures because it is the only one to have all of the following advantages. First, it is effective in gastrointestinal tract tumors where it is used first line for colorectal [28], small bowel [29] and stomach carcinomas [30]. Second, it can be used by the aerosol route [23].

Third, high doses of oxaliplatin given by the intraperitoneal route [9] are well tolerated [25]. The oxaliplatin dosage currently used during PIPAC procedures is $92 \mathrm{mg} / \mathrm{m}^{2}$, very close to the dosage used in intravenous infusions [23]. Local administration of oxaliplatin limits systemic toxicities because the peritoneum acts as barrier. The oxaliplatin dosage can be five times higher (460 $\left.\mathrm{mg} / \mathrm{m}^{2}\right)$ than the systemic dosage $\left(90 \mathrm{mg} / \mathrm{m}^{2}\right)$ for a similar serum level [25]. The time course and concentration of 
oxaliplatin in the peritoneal fluid and in the peripheral blood are available [25]. Safety data from large clinical studies indicate that the dose of intraperitoneal oxaliplatin with satisfactory tolerance is $300 \mathrm{mg} / \mathrm{m}^{2}[25,31]$ This dose which is lower than the dose limiting toxicity determined in the phase I study limits postoperative intraabdominal bleeding [31]. Clinical experience and safety monitoring have also been obtained with this high intraperitoneal dosage. We can therefore hypothesize that high-dose oxaliplatin given by intraperitoneal nebulization is feasible and safe. Furthermore, high doses of oxaliplatin improve drug efficacy. Oxaliplatin has dosedependent efficacy [32]. High-dose drug administration in intraperitoneal sites results in higher intratumoral concentrations, the physiopathological basis of greater efficacy. Furthermore, as decreased drug accumulation is probably one of the mechanisms underlying resistance to systemic treatments [33], the higher intratumoral concentration could prevent the development of systemic resistance. Importantly, oxaliplatin is a low cost generic drug. The costs of new antineoplasic drugs represent the fastest rising costs in Western health care and developing low-cost innovations is becoming a key strategic issue to preserve public health care insurance.

Author contributions: All the authors have accepted responsibility for the entire content of this submitted manuscript and approved submission.

Research funding: This study was funded by a PHRC-K grant (Programme Hospitalier de Recherche CliniqueCancer).

Employment or leadership: None declared.

Honorarium: None declared.

Competing interests: The funding organization(s) played no role in the study design; in the collection, analysis, and interpretation of data; in the writing of the report; or in the decision to submit the report for publication.

\section{References}

1. Jemal A, Bray F, Center MM, Ferlay J, Ward E, Forman D. Global cancer statistic. CA Cancer J Clin. 2011;61:69-90.

2. Jéhannin-Ligier K, Dantony E, Bossard N, Molinié F, Defossez G, Daubisse-Marliac L, et al. Projection de l'incidence et de la moratlité par cancer en France métropolitaine en 2017. Rapport technique. Saint-Maurice: Santé publique, 2017. 80.

3. Quere P, Facy O, Manfredi S, Jooste V, Faivre J, Lepage C, et al. Epidemiology, management, and survival of peritoneal carcinomatosis from colorectal cancer: A population-based study. Dis Colon Rectum. 2015;58:743-52.
4. Seyfried F, Von Rahden BH, Miras AD, Gasser M, Maeder U, Kunzmann $\mathrm{V}$, et al. Incidence, time course and independent risk factors for metachronous peritoneal carcinomatosis of gastric origin-A longitudinal experience from a prospectively collected database of 1108 patients. BMC Cancer. 2015;15:73.

5. Chia CS, You B, Decullier E, Lorimier G, Abboud K, Bereder JM, et al. Patients with peritoneal carcinomatosis from gastric cancer treated with cytoreductive surgery and hyperthermic intraperitoneal chemotherapy: Is cure a possibility? Ann Surg Oncol. 2016;23:1971-9.

6. Goéré D, Malka D, Tzanis D, Gava V, Boige V, Eveno C, et al. Is there a possibility of a cure in patients with colorectal peritoneal carcinomatosis amenable to complete cytoreductive surgery and intraperitoneal chemotherapy? Ann Surg. 2013;257:1065-71.

7. Levine EA, Stewert JH, IV, Shen P, Russell GB, Loggie BL, Votanopoulos KI. Intraperitoneal chemotherapy for peritoneal surface malignancy: Experience with 1,000 patients. J Am Coll Surg. 2014;218:573-85.

8. Liu Y, Yonemura Y, Levine EA, Mizumoto A, Hirano M, Sako S, et al. Cytoreductive surgery and hyperthermic intraperitoneal chemotherapy for peritoneal dissemination from small bowel malignancy: Results from a single specialized center. Ann Surg Oncol. 2016;23:1625-31.

9. Elias D, Lefevre JH, Chevalier J, Brouquet A, Marchal F, Classe $J M$, et al. Complete cytoreductive surgery plus intraperitoneal chemohyperthermia with oxaliplatin for peritoneal carcinomatosis of colorectal origin. J Clin Oncol. 2009;27:681-5.

10. Franko J, Shi Q, Goldman CD, Pockaj BA, Nelson GD, Goldberg RM, et al. Treatment of colorectal peritoneal carcinomatosis with systemic chemotherapy: A pooled analysis of north central cancer treatment group phase III trials N9741 and N9841. J Clin Oncol. 2012;30:263-7.

11. Sadeghi B, Arvieux C, Glehen O, Beaujard AC, Rivoire M, Baulieux J, et al. Peritoneal carcinomatosis from non-gynecologic malignancies: Results of the EVOCAPE 1 multicentric prospective study. Cancer. 2000;88:358-63.

12. Razenberg LG, Van Gestel YR, Lemmens VE, De Hingh IH, Creemers GJ. Bevacizumab in addition to palliative chemotherapy for patients with peritoneal carcinomatosis of colorectal origin: A nationwide population-based study. Clin Colorectal Cancer. 2016;15:41-6.

13. Shirao K, Boku N, Yamada Y, Yamaguchi K, Doi T, Goto M, et al. Randomized phase III study of 5-fluorouracil continuous infusion vs. sequential methotrexate and 5 -fluorouracil therapy in far advanced gastric cancer with peritoneal metastasis (JCOG0106). Jpn J Clin Oncol. 2013;43:972-80.

14. Thomassen I, Van Gestel YR, Van Ramshorst B, Luyer MD, Bosscha K, Nienhuijs SW, et al. Peritoneal carcinomatosis of gastric origin: A population-based study on incidence, survival and risk factors. Int J Cancer. 2014;134:622-8.

15. Chua TC, Yan TD, Saxena A, Morris DL. Should the treatment of peritoneal carcinomatosis by cytoreductive surgery and hyperthermic intraperitoneal chemotherapy still be regarded as a highly morbid procedure?: A systematic review of morbidity and mortality. Ann Surg. 2009;249:900-7.

16. Glehen O, Gilly FN, Boutitie F, Bereder JM, Quenet F, Sideris L, et al. Toward curative treatment of peritoneal carcinomatosis from nonovarian origin by cytoreductive surgery combined with 
perioperative intraperitoneal chemotherapy: A multi-institutional study of 1,290 patients. Cancer. 2010;116:5608-18.

17. Jacquet $\mathrm{P}$, Sugarbaker $\mathrm{PH}$. Clinical research methodologies in diagnosis and staging of patients with peritoneal carcinomatosis. Cancer Treat Res. 1996;82:359-74.

18. Goéré D, Souadka A, Faron M, Cloutier AS, Viana B, Honoré C, et al. Extent of colorectal peritoneal carcinomatosis: Attempt to define a threshold above which HIPEC does not offer survival benefit: A comparative study. Ann Surg Oncol. 2015;22:2958-64.

19. Coccolini F, Catena F, Glehen O, Yonemura Y, Sugarbaker PH, Piso $\mathrm{P}$, et al. Complete versus incomplete cytoreduction in peritoneal carcinosis from gastric cancer, with consideration to PCI cut-off. Systematic review and meta-analysis. Eur J Surg Oncol. 2015;41:911-9.

20. Mogal H, Chouliaras K, Levine EA, Shen P, Votanopoulos KI. Repeat cytoreductive surgery with hyperthermic intraperitoneal chemotherapy: Review of indications and outcomes. J Gastrointest Oncol. 2016;7:129-42.

21. Esquis $P$, Consolo D, Magnin G, Pointaire P, Moretto P, Ynsa $M D$, et al. High intra-abdominal pressure enhances the penetration and antitumor effect of intraperitoneal cisplatin on experimental peritoneal carcinomatosis. Ann Surg. 2006;244:106-12.

22. Solaß W, Hetzel A, Nadiradze G, Sagynaliev E, Reymond MA. Description of a novel approach for intraperitoneal drug delivery and the related device. Surg Endosc. 2012;26:1849-55.

23. Demtröder C, Solass W, Zieren J, Strumberg D, Giger-Pabst U, Reymond MA. Pressurized intraperitoneal aerosol chemotherapy (PIPAC) with oxaliplatin in colorectal peritoneal metastasis. Colorectal Dis. 2016;18:364-71.

24. Nadiradze G, Giger-Pabst U, Zieren J, Strumberg D, Solass W, Reymond MA. Pressurized intraperitoneal aerosol chemotherapy (PIPAC) with low-dose cisplatin and doxorubicin in gastric peritoneal metastasis. J Gastrointest Surg. 2016;20:367-73.
25. Elias D, Bonnay M, Puizillou JM, Antoun S, Demirdjian S, El OA, et al. Heated intra-operative intraperitoneal oxaliplatin after complete resection of peritoneal carcinomatosis: Pharmacokinetics and tissue distribution. Ann Oncol. 2002;13:267-72.

26. Dindo D, Demartines N, Clavien PA. Classification of surgical complications: A new proposal with evaluation in a cohort of 6336 patients and results of a survey. Ann Surg. 2004;240:205-13.

27. Common Terminology Criteria for Adverse Events v 4.0 (CTCAE) http://ctep.cancer.gov/

28. Douillard JY, Siena S, Cassidy J, Tabernero J, Burkes R, Barugel M, et al. Final results from PRIME: Randomized phase III study of panitumumab with FOLFOX4 for first-line treatment of metastatic colorectal cancer. Ann Oncol. 2014;25:1346-55.

29. Xiang XJ, Liu YW, Zhang L, Qiu F, Yu F, Zhan ZY, et al. A phase II study of modified FOLFOX as first-line chemotherapy in advanced small bowel adenocarcinoma. Anticancer Drugs. 2012;23:561-6.

30. Cunningham D, Starling N, Rao S, Iveson T, Nicolson M, Coxon $\mathrm{F}$, et al. Capecitabine and oxaliplatin for advanced esophagogastric cancer. N Engl J Med. 2008;358:36-46.

31. Pomel C, Ferron G, Lorimier G, Rey A, Lhomme C, Classe JM, et al. Hyperthermic intra-peritoneal chemotherapy using oxaliplatin as consolidation therapy for advanced epithelial ovarian carcinoma. Results of a phase II prospective multicentre trial. CHIPOVAC study. Eur J Surg Oncol. 2010;36:589-93.

32. Maindrault-Goebel F, De Gramont A, Louvet C, André T, Carola E, Gilles $\mathrm{V}$, et al. Evaluation of oxaliplatin dose intensity in bimonthly leucovorin and 48-hour 5-fluorouracil continuous infusion regimens (FOLFOX) in pretreated metastatic colorectal cancer. Oncology multidisciplinary research group (GERCOR). Ann Oncol. 2000;11:1477-83.

33. Alcindor T, Beauger N. Oxaliplatin: A review in the era of molecularly targeted therapy. Curr Oncol. 2011;18:18-25. 